\title{
Understanding Multipath Packet Scheduling for Future IoT
}

\author{
Nikhil Reddy Gade, Deakin University, Burwood, Australia \\ Email: ngade@deakin.edu.au
}

\begin{abstract}
Future loT systems like WiGig or $5 G$ usages for Industrial loT has led to a growth of Internet of Things and Cyber Physical Systems in the industry and has proven evident already. Automation with information trade inside and among manufacturing plants is going through a huge change alongside IloT, which is made conceivable by the continuous combination of data innovation (IT) with operational innovation (OT). Such an assembly can be possibly profited by together utilizing ongoing advances in correspondence innovations, e.g., coinciding WiGig and 5G. In this undertaking, we initially examine the current specialized difficulties for the concurrence of WiGig and 5G for IT/OT combination, at that point create and assess a hearty multipath convention (drawing on WiGig and 5G flawlessly) to make ultrareliable and smooth network. The undertaking will give a unique design to the future mechanical correspondences, interfacing modern robots ideal, investigating information exactly and conveying constant bits of knowledge dependably.
\end{abstract}

\section{Introduction}

The wireless links are the most important aspects if this bandwidth aspects as all the multipath systems are transferred with wireless links. The wireless links are used in this transformation is used for the information from one point to another point as routes of these multiple layers are undertaken in this transformation. The multiple points are used in these interlined layers and every layer has the capability in order to execute the bandwidth in the right way so that there won't be any delay in the transformation of information one place to another place.

For this reason, it has been understood that bandwidth is the aspect which needs to be undertaken by the various interlinked layers. The various layers are experimented in this field that need to be enhanced as the required speed to transfer the knowledge from one place to another place in the wonderful way. The need of bandwidth with multiple paths were upgraded with the involvement of TCP that has been undertaken by number of interlinked layers. S. Floyd et al. [1] said these are the layers that need to be takes as the creative implementation because of the performance that has been assured with the wireless implementation that enriched the experience of the users in the field of multiple bandwidth paths. The usage of the electronic device has been very popular in every corner of the world as the interface development become the most important aspect for the promotion of electronic device. The electronic devices have been equipped wonderfully with various interfaces that have been helped to undertake the networks like and 3G and 4G. D. Murray et al. [2] stated that the devices have been introduced with multiple interfaces that is able to give utmost quality to the devices which are used with multiple devices. These devices have been used or the various purposes but there are certain issues of quality which is associated with the multiple interfaces.

The quality has been improved initially with the help of the bandwidth consumption with various multimedia acts and games which are introduced with real time nature. The usage of multiple bandwidth systems has been seen in most of the interfaces but the most important aspect of this system it is not supported by legacy which is known as TCP. The experiments have been undertaken wonderfully in order to extend operations of TCP and undertake the multipath TCP.

The concurrent and compatibility of multiple phases of these interfaces have been in the operations of this MTCP related aspects. The entire data which is used in this MTCP is split into different parts and all these 
parts are sent to the right user within the short span of time. The shortest span of time is used in this multipath because of the various interfaces that are used in this process of transforming from one point of contact to another point of contact [3]. The sub flow is the most important aspect of multipath. Every flow of transformation of information from one place to another place is absolutely possible with the implementation of various bandwidth implementations. The application layer is most important layer used in development of network layer.

\section{Aims}

The aim of this paper is to understand the importance of TCP and MPTCP in regard of ensuring networking speed. Studying the technology that gives compatibility of using the data has been done in this paper and to solve the bottleneck congestion problem for the MPTCP to increase the overall efficiency. This is done by applying the SB_CC (Shared Bottleneck based Congestion Control scheme) algorithm over MPTCP.

\section{Scope of the paper}

The multiple path bandwidths have been developed with the help of the MPTCP as is has leveraged the performance of multiple paths. The performance improvement is assured in regard of the MPTCP and diversity of information which is transferred between two different points is seen in this MPTCP implementation. Shen et al. [4] stated the MPTCP is highly used in the cellular related devices as most of the devices that are used in the usage of MPTCP is correlated with the cellular devices. The most common things that can be seen in the usage of this MPTCP relate devices is the wireless routes that are used in transformation of different kinds of interfaces. The tolerant multipath system is introduced recently in order to control the congestion that is used in the MPTCP. The congestion control is very important as the network traffics are very common when the data is transferred from one place to another place. The route heterogeneity is the important reason for most of the buffer which takes place in the usage of network systems. The network systems need to be controlled with the brand-new congestions as these congestions are capable enough in solving various buffer related issues that takes place while streaming various videos at the internet [5].

\section{List of research questions:}

- What are the important features that are associated with the MPTCP and TCP?

-What role does MPTCP play in assuring the quality of networking in most of the areas where speed of the network systems is highly needed?

- Discussing on the other technologies that involved in supporting the usage of the MPTCP and TCP networking systems.

-What are experiments that have been undertaken in order to increase the efficiency of the TCP networking systems in organizations?

- Explain the role of IoT in the organizations which are turning their operations towards the implementation of TCP and MPTCP in the operations of organizations which are executing their operations with IT related networking systems? 
- How to understand the challenges which are faced by the users in turning out their systems towards the usage of TCP and MPTCP in the operations of various businesses that are involved in TCP functions?

\section{Sources for research questions:}

- The first important source that has been used for collecting the information in this paper is reviewing various articles that are written on TCP technology.

- Handley et al. [6] stated that there are several books written specifically regarding the development of loT technology that led to the revolutionary inventions in the field of the technology that is based on the networking systems.

- Various Videos which are related to the implementation of advanced technology has been seen in the operations of various businesses in the organizations.

- There are even certain news articles which are used for the development of this paper.

\section{Data collection:}

The data for this work is collected BY using the. MAHIMI light well tools, which has command like delay shell, link shell and loss shell and ns3 simulator. From this we are going to calculate the final state and average round trip time as features and the target variable.

\section{Conceptual framework of TCP and MPTCP:}

This can be solved to the great extent if the MPTCP systems are interlinked with congestion control tools which have good impact on the stoppage of buffer that takes place in the implementation of this bandwidth in the usage of various networks. In the implementation of the TCP and MPTCP the best aspect that need to be concentrated is identification of the areas where generally buffer takes place. The areas which are responsible for buffer and loss of channel in the midst of browsing various websites can be investigated in the right way. The investigation can be undertaken by with the loss of wireless connections figure 1 explains Multipath TCP

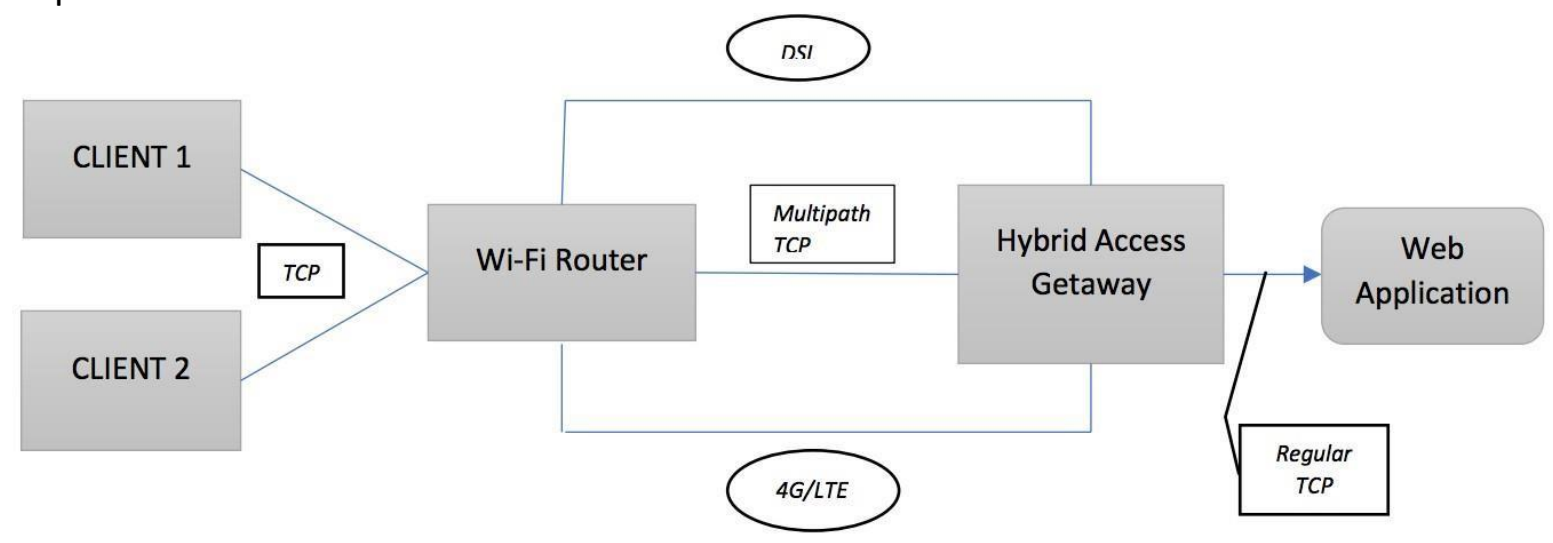

Figure 1: Multipath TCP 
The areas where these wireless connections are broken can be found out very easily in regard of the systems are used and implemented with MPTCP. Barré et al. [8] said in this way it has been understood that buffer related issues are highly reduced with the implementation of MPTCP in the wireless areas. The conventional TCP is associated with the MPTCP as the large bandwidth networks were implemented in this multipath system. The TCP needs to be checked and confirmed in the right way in order to overlook the buffer issues that can be reduced with the help of TCP that is associated with MPTCP. The core problem which is associated with the TCP implementation is the multiple interface system that is increased with the implementation and usage of the devices in the short span of time. The devices which are used for the interfaces that are implemented in the transfer of information from one place to another place has been recognized [9].

The TCP usage and load has been developed greatly that lead to the low performance of the interfaces which are used in the devices. The coupled congestions and algorithms are used in the MPTCP implementation that gives absolute possibility of congestion control. This has enabled the implementation of the MPTCP in various aspects of data transformation. The fluid models are highly used in regard of the coupled congestion. The coupled congestion are the areas where every bandwidth association are interlinked. The algorithms are used mostly for the reason of improving the quality of the streaming and sending data from one place to another place. The TCP and MPTCP is coupled with various algorithms.

The important challenge that has been seen in regard of this TCP and MPTCP is establishing friendliness and responsive behavior among the algorithms that are used for the connection between TCP and MPTCP. The MPTCP algorithm is used in most of the bandwidth are highly friendly and able to control the network systems that are leading to the multiple paths. Mirani et al. [10] claims the important reason for the implementation of the MPTCP algorithm is responsiveness of bandwidth which is used in this network system. The connection between TCP and MPTCP need to be analyzed in the right way in order to dominate the buffer that is appearing while using the traditional systems. There is the need of the expansion of this kind of systems in order to give the best performance to the users who are involved in the enhancement of the data and network transferring from place to another place. The responsiveness of this MPTCP in most of the cases was very low that has made the interlink between the TCP and MPTCP has less friendly.

The comparison needs to be solved to the great extent in order to reduce the losses which have been identified in the usage of the MPTCP that is correlated with the usage of the TCP and interconnection. The users who are undertaking the operations of this TCP and MPTCP are very happy when they are used individually. This is because these two network systems are working wonderfully if they are used individually but, in those cases, where these two are interlinked with each other have destroyed usage of this interlink connection [11]. Because of all these reasons it has been identified that there is the need of make the experiment that can help the users to enjoy the usage of both network the systems. The network systems can be ensured their design in such a way that blend of both TCP and MPTCP. The quality assurance should be the most important aspect that need to be interlinked with the usage of TCP and MPTCP as the speed and other characteristics are interested by most of the users who are depending entirely in these two network systems in transfer of data from one point to another point. The speed of connecting the devices with Wi-Fi is increased when TCP and MPTCP has been increased compare to other traditional systems. S. R. Pokhrel et al. [12] said in this way it has been understood that every user needs to understand the importance of this TCP and MPTCP implementation. 
The usage of this Wi-Fi has been seen in most of the device usage in the recent days because of the wireless advantages that are used in this usage. The MPTCP is used for the channel establishment and independent channel is used for the purpose of sharing the network. But the process of sharing the networks using Wi-Fi has led to the collusion of networks between two or more networks. Every day the usage of these networks and sharing of the networks has been increased tremendously which has made the situation worse. The impact of this collusion leads to low demand on the Wi-Fi network which needs to make the developed operations in the field of this networks. Khalili et al. [13] states networks which are associated with the WiFi has to be enhanced with interfaces that can match characteristics of the TCP and MPTCP. For that reason, it is very important for increasing the experiments that can enhance the usage of the Wi-Fi operations that are associated with the TCP and MPTCP operations.

This made the networking very complex and network line development is highly necessary for the development of every operation that can enhance the usage of TCP and MPTCP in regard of the Wi-Fi operations. The cellular network routes are decreased to the great extent with the implementation of Wi-Fi networking because of the various advantages that are associated with the Wi-Fi usage. The networks which are routed with the help of cellular networks need0 to be controlled to the great extent. For that reason, it has been understood that Wi-Fi has the greater popularity than the other networking systems. It has also been observed that buffer time is very high in the cellular networks. The Wi-Fi is the networking system that has the capability of reducing the buffer to the great extent. The data packets are assured with the MPTCP which is absolutely standardized with IETF. S. R. Pokhrel et al. [14] states that the MPTCP is valued with highspeed packets. The speed which is associated with data packets has been developed because of the highspeed links that are known as AP and BS.

The one-way connection was interrupted with the AP link but in order to reduce this kind of interruptions there is the need of combining the AP and BS. In this way even the data packets are able to give best networks with amazing speed. The speed can be reduced once the data is completed. The MPTCP routes can be enhanced with the help of MPTCP LIA where there is the establishment of different congestion windows were established. S. R. Pokhrel et al. [15] explains the updated version of MPTCP that has implemented in the organization that enhances the outcome of the networks that is associated with the implementation of MPTCP LIA. In this way there is the need of the implementation of MPTCP LIA especially in the areas of data packets.

The data packets are the areas which make the individual users comfortable in accessing the data wherever they visit. In this way it can be said that MPTCP is the best way of offering data packets. The devices have been introduced with multiple interfaces that is able to give utmost quality to the devices which are used with multiple devices. These devices have been used or the various purposes but there are certain issues of quality which is associated with the multiple interfaces. The quality has been improved initially with the help of the bandwidth consumption with various multimedia acts and games which are introduced with real time nature. The usage of multiple bandwidth systems has been seen in most of the interfaces but the most important aspect of this system it is not supported by legacy which is known as TCP [16].

\section{Description and implementation of these operations}

The critical industrial automation has been seen as the important challenge that has been faced by most of the companies and solving these challenges had turned out to be most important aspect. The automation has been possible with the implementation of loT systems in the operations of the business. The 
standardization of the IT convergence has become the crucial factor that is helping to address challenges in move towards convergence process. The automation process is needed in almost every area of the IT. The advanced practice of $6 \mathrm{TiSCH}$ has been enhanced in most of the areas where IT related works are executed. For that purpose, it is very important for making all the devices which are used in the IT field need to be interlinked with the IoT which increased the quality of the networks which are used in the place were IT implementations are highly used [17].

\section{Evaluation of the network systems}

The notion of the MPTCP is helped to connect multiple networks from various fields. The systems which are used in this MPTCP can give high privilege in giving wonderful networking system that can help the users to increase their knowledge in regard of implementation of MPTCP in regard of IloT. IloT requirement was increased to the great extent which assures major characteristics like come Application MPTCP Sub flow TCP1 Sub flow TCPn IP Application layer Transport layer Network Layer latency and energy efficiency. The energy with which the networking services offered in regard of IloT has been seen in the segregating of different aspects of network systems. M. Pittoni et al. [18] explains that the peculiar stoppage of networking has been seen in the usage of this IloT systems in the operations of various businesses. This has made most of the people to opt this IIoT systems in the operations of the businesses activities which are executed by using these sorts of networking systems. This can be seen in most of the organizations as they have reaping the advantages.

The resisting power of network interruptions is seen in the usage of MPTCP is establishment of control stations that can solve the issues that involved in the usage of loT systems. The $4 \mathrm{G}$ link has also been interlinked with these systems where the speed of using the devices can be increased to the great extent. The loT systems that are established in the networking organizations have tremendous results which made most of the organizations to give utmost speed to the users who are involved in the implementation of $4 \mathrm{G}$ network systems. The downloading is the best aspect that is associated with the implementation of the MPTCP based cellular networks in the organizations. The MPTCP is the network system that is highly used in most of the areas in order to develop the speed of downloading and intricate the usage of the users who are involved in its usage [19].

The cellular routes for establishing the best routes in the operations of the MPTCP and assuring the best downloading is absolutely possible with the networking systems established in the organizations. The downloading is possible even in the small phones where data packets are offered to the people who involved themselves to use these packets. The compatibility is the most important aspect of this MPTCP systems. The TCP is considered as the wonderful invention that helped millions of users to access whatever the information they need from various sources. This made the users to use this MPTCP and TCP networking systems that give best service to the users who are seriously working on the transformation of data from one place to another place. Thus, it has been understood that MPTCP is the important study that need to be studied in order to enhance the importance of networking systems [20].

\section{Risks of TCP and MPTCP implementation:}

1. Computer virus: Computer viruses have been in the news lately for the devastating network security risks they've caused around the world this year. Those are instances of virus attacks on a global scale, but viruses can pose just a big of a threat to smaller companies. Viruses can corrupt your files and delete valuable data, negatively impacting your daily operations. At their worst, viruses have the potential to wipe clean your entire hard drive. As such, it's important to advise your staff to never open an email or click on a link they don't recognize. 
2. Software Vulnerabilities Pose Network Security Risks: When a security update pops up in the midst of a busy day, it's easy click out of it and then forget to come back to it later. But if you're not updating your software as regularly as you should, you could be putting your company at risk. Alay et al.

[21] states that the outdated software can eventually slow your entire network down to the point where you can't get work done. It could cause your site to crash, and while it's down, you could be losing customers. If you're an entrepreneur or small business, that could be a major issue.

3. Hackers: We often think of networks security risks as things that live in our computers. But computer hackers can cause devastating damage from anywhere. Once hackers break into your system, they have free rein. They can steal files, gain access to privileged information or expose trade secrets for your competitors and the world to see. Hackers may be on the other side of the planet, but if you value your network security, they shouldn't be out of your mind.

4. You Don't Know What You Don't Know: As technology evolves, network security risks become more sophisticated too. When you're doing all other things, it takes to make a company successful, keeping up with network security can fall through the cracks. You may not be aware of a problem until it's too late. This lack of education and focus on what can be such a major issue can really hurt your company down the line. Don't be afraid to call in the experts and outsource your network security to the experts who dedicate all their time to fighting these risks

\section{Research methodology:}

They are several methods to improve the efficiency of MPTCP. According to S. R. Pokhrel and S. Garg, the data traffic in industry of $4 \mathrm{~g}$ is complex and multi-dimensional. So, the algorithm we have to use must perform the sequential operations in this case it is better with the implementation of reinforcement learning. Which can be further enhanced by implementing the deep neural network AS:

1) DQN adopts experience replay which uses stochastic optimization this can help to get more accuracy and to be exact word precision

2) DQN utilizes target network. This is because it estimates and changes it values based on the target which is obtained for the no of steps calculated from the reply buffers. And, finally as in the all reinforcement learning we use the SoftMax function as part of optimization for the algorithm of deep q network. Below you can see the algorithm which we run for the first sprint.

The other method as proposed by (Ton-Wenjia) by applying the SB_CC (Shared Bottleneck based Congestion Control scheme) algorithm which solves the main issue faced. 


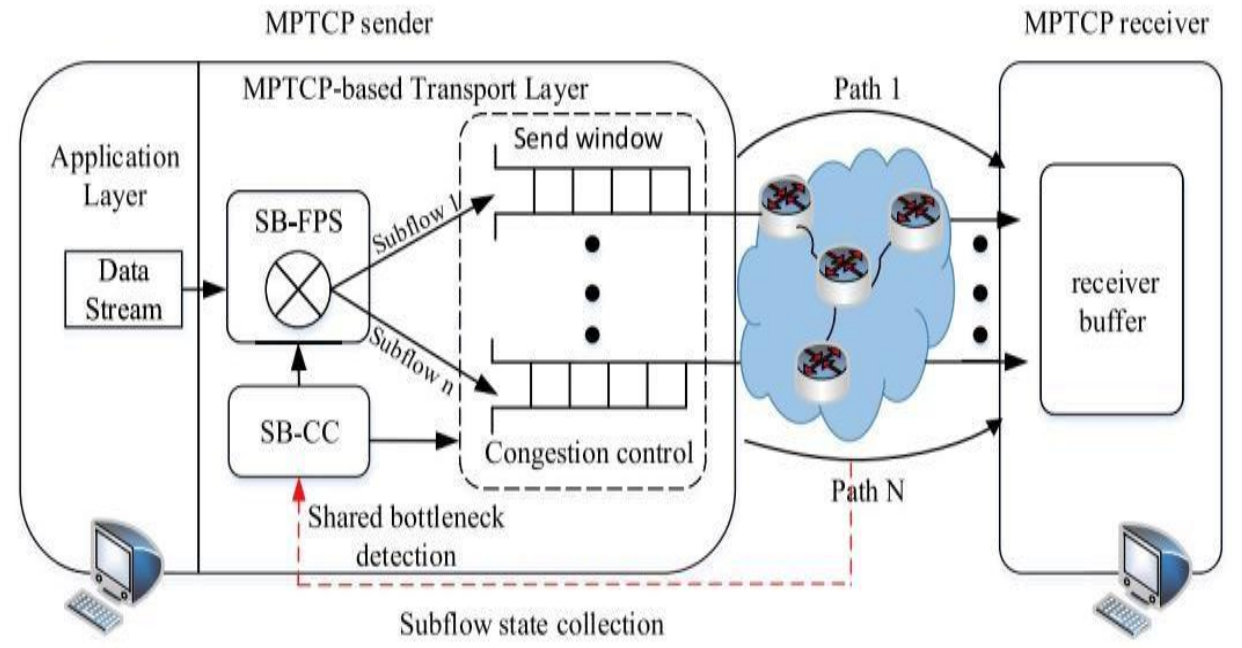

Fig. 1. Framework of the proposed scheme. (1) SB-CC groups the subflows into different bottleneck set according to the state of each subflow. The subflows in the same bottleneck are coupled controlled. (2)Based on the information provided by SB-CC, SB-FPS models each subflow's behavior and estimates the number of packets scheduled to each subflow. 
First sprint:

Our Aim in the first sprint is to choose the best algorithm and write the python code, this algorithm helps us to implement efficiently MPTCP and finally this is evaluated in Linux kernel.

Algorithm 1 DQL-MTCP Algorithm

1: procedure INITIALIZE

2: Network Representation $\mathbb{N}($.$) , Actor \mathbb{E}($.$) and Critic \mathbb{C}($.

3: Target Networks $\hat{\mathbb{N}}(.) \hat{\mathbb{E}}(.) \mathbb{C}($.

4: Ornstein-Uhlenbeck process $\partial$

5: end procedure

6: procedure CONGESTION CONTROL

7: While (window Adapt) do

8: Compute final state $\mathbb{F}($.$) using \mathbb{N}($.

9: Compute update action $\hat{\boldsymbol{a}}(t)$ from Actor $\mathbb{E}\left(\boldsymbol{f}^{N}(t)\right)$

10: Generate action $\boldsymbol{a}(t)$ using process $\partial$ and $\hat{\boldsymbol{a}}(t)$

11: Observe $\mathbb{R}($.$) with \boldsymbol{a}(t)$

12: Save transitions $(\mathbb{S}(t), \boldsymbol{a}(t), \mathbb{R}(t), \mathbb{S}(t+1))$ in to Memory

13: procedure TRAINING

14: Sample $k$ transistions $\left(\mathbb{S}_{i}, \boldsymbol{a}_{i}, \mathbb{R}_{i}, \mathbb{S}_{i+1}\right)$ from Memory

15: Compute $\boldsymbol{f}_{i+1}^{N}$ using $\hat{\mathbb{N}}\left(\mathbb{S}_{i+1}\right)$

16: Evaluate target for Critic $\mathbb{C}(),. y_{i}=\mathbb{R}_{i}+\gamma \hat{\mathbb{C}}\left(f_{i+1}^{N}, \hat{\mathbb{E}}\left(f_{i+1}^{N}\right)\right)$

17: Critic parameters $\min ($ loss $), 1 / k \sum_{i}^{k}\left(y_{i}-\mathbb{C}\left(\boldsymbol{a}_{i}, \boldsymbol{f}_{i}^{N}\right)\right)^{2}$

18: Compute Policy Gradient from Critic: $\nabla_{a} \mathbb{C}\left(\boldsymbol{a}_{i}, \boldsymbol{f}_{\boldsymbol{i}}^{\boldsymbol{N}}\right)$

19: Update actor parameters, $1 / k \sum_{i}^{k} \nabla_{a} \mathbb{C}\left(\boldsymbol{a}_{i}, \boldsymbol{f}_{i}^{N}\right) \cdot \nabla_{k} \mathbb{E}\left(\boldsymbol{f}_{i}^{N}\right)$

20: Compute Policy gradient from Actor: $\nabla_{k} \mathbb{E}\left(f_{i}^{N}\right)$

21: Update network representation parameters (policy gradient), $1 / k \sum_{i}^{k} \nabla \boldsymbol{a} \mathbb{C}\left(\boldsymbol{a}_{i}, \boldsymbol{f}_{i}^{N}\right) \cdot \nabla k \mathbb{E}\left(\boldsymbol{f}_{i}^{N}\right) \cdot \nabla_{\mathbb{R}_{i}} \mathbb{R}\left(\mathbb{S}_{i+1}\right)$.

22: $\quad$ end procedure

23: procedure UPDATE TARGET NETWORK

24: $\hat{\mathbb{N}}():.=.001 \mathbb{N}()+..999 \hat{\mathbb{N}}(.) ; \hat{\mathbb{E}}():.=.001 \mathbb{E}()+..999 \hat{\mathbb{E}}($.$) ;$ $\hat{\mathbb{C}}():.=.001 \mathbb{C}()+..999 \hat{\mathbb{C}}($.

25: $\quad$ end procedure

26: endWhile

27: end procedure

\section{FIG: Algoritham for DQL_MPTCP}

This algorithm is designed by (S. R. Pokhrel and S. Garg)

DQL-based multipath congestion control and scheduling mechanism is illustrated in the Algorithm. DQL-MPTCP algorithm initializes the parameters of Representation Network N(.), Critic networks $Q($.$) and Actor networks$ $E\left(\right.$.). In order to ensure stable learning, we have to use target networks $N^{\wedge}\left(\right.$.) $E^{\wedge}\left(\right.$.) $Q^{\wedge}($.$) , which replicate the$ structure of their corresponding networks, i.e., $\mathrm{N}($.$) , Actor \mathrm{E}($.$) and Critic \mathrm{Q}($.$) respectively.$

For the intermingling of the learning cycle, the target network parameters are refreshed utilizing a smallish control parameter with the end goal that the target parameters are adjusted gradually in every iteration review that the target network, by plan, requirements to update gradually for stability. Our MPTCP DQL agent runs constantly, tuning in for periodic questions from the MPTCP source (executed in the kernel)

S. R. Pokhrel et al. [22] states that to enable investigation, which is useful when preparing inexperienced DQL agent, we add corresponded noise, utilizing the Ornstein-Uhlenbeck stochastic interaction to control actions in 
run-time. Such coupling of focused on experience replay with investigation helps the DQL specialist improve its actions of changing subflow window sizes and packet scheduling.

The final state network representation of all active subflows $F($.$) is derived from the representation network$ $\mathrm{N}\left(\right.$.) (see step 8, Algorithm), and the control action for the target MPTCP subflow $\mathrm{a}^{\wedge}(\mathrm{t})$ is computed by using the actor $\mathrm{E}().[22]$.

The second algorithm we are going to decide is SB_CC which solves the congestion problem

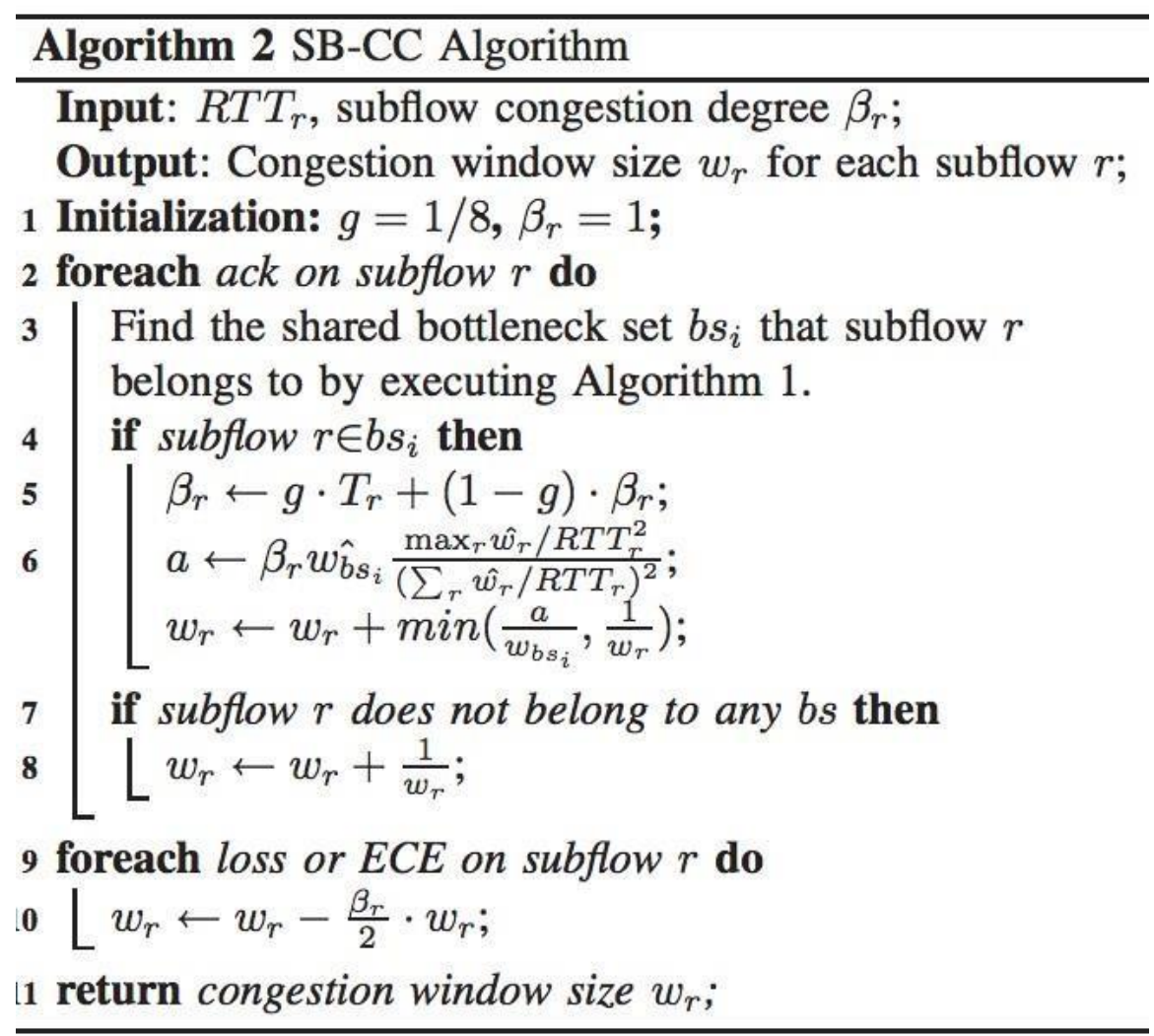

Wei, $W$ et al. [24] explained in detail that $\lambda_{r}$ is the congestion loss rate on subflow $r$. Furthermore, we can compute a from Eq. (4):

$\operatorname{Min}\left(\frac{\mathrm{a}}{w^{\wedge} \mathrm{bs}}, \frac{1}{w_{r}}\right)\left(1-\lambda_{r}\right)=\frac{\beta \mathrm{r}}{2} w_{r}^{\wedge} \lambda_{r}$.

Since $\lambda_{r}$ is usually very small, i.e., $1-\lambda_{r} \approx 1$. from Eq. (4), and $w_{r}{ }^{\wedge \mathrm{TCP}}=\sqrt{\frac{2}{\lambda_{r}}}$, subflow $r$ increases the window size $w_{r}$, and the corresponding aggressiveness factor a can be computed as: $w_{r}=w_{r}+\min \left(\frac{a}{w_{b s}}, \frac{1}{w_{r}}\right)$, $\mathrm{a}=\beta_{r} w_{b s} \wedge \frac{\max _{r} w_{r} \wedge / \mathrm{RT} T_{r} T_{r}}{\left(\frac{\sum \mathrm{r} w_{r}}{\mathrm{RTTr}}\right)^{2}}$. Algorithm SB-CC is shown in Algorithm 2. By jointly considering subflows in one bottleneck set, the proposed SB-CC can achieve the goal of "do no harm" to other TCP connections sharing the same bottleneck and mean while improve the overall network performance. To be noted, our proposed SB-CC can detect and maintain multiple bottlenecks sets for one MPTCP connections, and it also adapts to the dynamic changing scenarios of shared bottlenecks [24].

\section{Second sprint:}

In the second sprint of the artefact development, we are going to install virtual ubuntu then we are going to install the MAHIMI, finally we are going to install the TensorFlow package in the python library. And lastly, 
we are going to install the DQLMPTCP kernel. Then we are going to end this one with building server and client. So, in the second sprint of the artefact development what I have done is nothing but install all the software and tools required for implementation of DQLMPTCP where in the first sprint we have just designed the code for the algorithm, in the second sprint we are installing all the required applications for its implementation.

\section{Third sprint:}

At first sprint we have developed the algorithm for the congestion control SS-BC proposed by Wenjia [24].

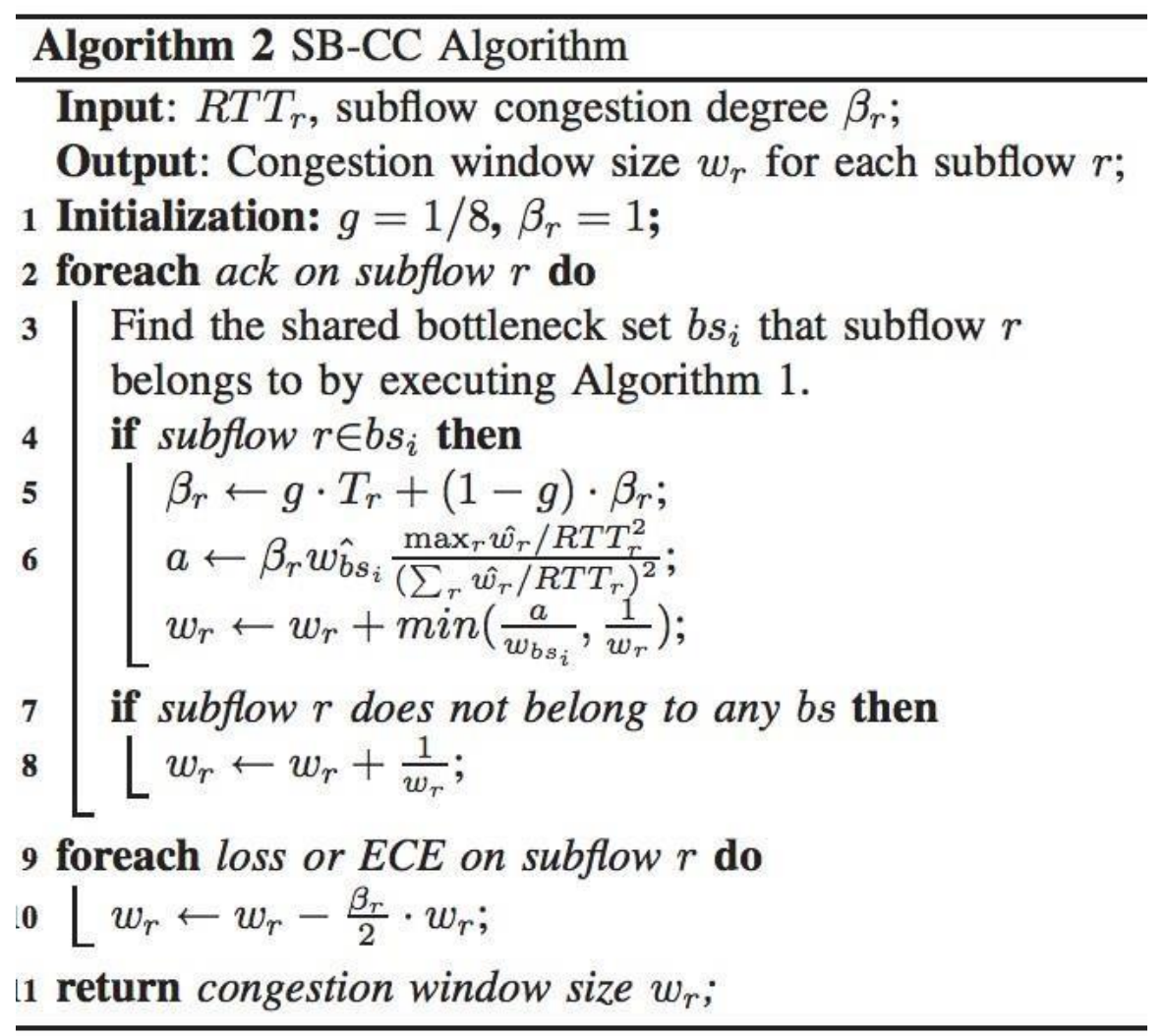

Fig: SS_BC (Shared Bottleneck based Congestion Control scheme) algorithm (ToN-WENJIA)

For the window reduction aspect of SB-CC, we define congestion degree, a term similar to DCTCP, which works with window reduction for each MPTCP subflow, similarly to DCTCP. Then, in order to achieve bottleneck fairness while maintaining stability, we leverage the defined congestion degree in restricted additive increase to produce coupled fine-grained bottleneck-fairness congestion control.

It should also be emphasized that our scheme is called RTT compensator. This is still a congestion control technique based on network fairness. Our suggested SBCC, on the other hand, is a bottleneck fairness based linked congestion management technique that may be used in a variety of scenarios give a better throughput than the current MPTC While maintaining the bottleneck, congestion control strategies are use fairness.

In the second sprint we have installed all the required applications and finally in the third sprint we are going to implement and evaluate our artefact. It is important to note that our designed artefact won't work for the time varying networks. In the third sprint we implemented our code python programming in the Unix shell environment by using the mahiminet So in general how algorithm works is nothing but DQN will collect 
information for each sub path runtime, it will interact periodically by updating the runtime information in each case of the congestion window. So basically, our features in the dataset we are going to use is final state for each sub flow, average round trip time this all be used for predicting the availability of sending time next slot which is our objective. So, the target variable is nothing but the prediction of the availability of sending rate for the next slot. The performance evaluation in this case is done by using the ns 3 simulator. Which is nothing but comparison of throughoutput of both TCP and MPTCP and our proposed algorithm. The new connectivity of the devices in the MPTCP and TCP is observed by how much empty space left in the reply buffer, based on this we calculate the no of extra devices added in the network for each framework.

\section{Evaluation Of artefact:}

We evaluate our model in ubuntu environment by creating the above algorithm in python code and running it UNIX environment as we know that it is difficult to evaluate this algorithm in the real-world scenario., So basically our features in the dataset we are going to use is final state for each sub flow, average round trip time this all be used for predicting the availability of sending time next slot which is our objective. So, the target variable is nothing but the prediction of the availability of sending rate for the next slot. The performance evaluation in this case is done by using the ns3 simulator. Which is nothing but comparison of throughoutput of both TCP and MPTCP.

\section{Conclusion:}

We know that the main problem with the MPTCP is congestion, to address this issue we suggested an algorithm proposed by (ToN_Wenjia) SB-CC which is used to solve the issue faced by the current MPTCP "Congestion control", from the literature review we also know that the algorithm SS-BC is a bottle neck fairness congestion control model.

\section{References:}

[1] S. Floyd, D. K. K. Ramakrishnan, and D. L. Black, The Addition of Explicit Congestion notification (ECN) to IP, document RFC 3168, IETF, 2013.

[2] D. Murray, T. Koziniec, S. Zander, M. Dixon, and P. Koutsakis, "An analysis of changing enterprise network traffic characteristics," in Proc. 23rd Asia-Pacific Conf. Commun. (APCC), Dec. 2017, pp. 1-

6.

[3] QoS: Congestion Avoidance Configuration Guide, Cisco IOS XE Release 3S. Accessed: Jan. 19, 2020. [Online]. Available: https://www.cisco.com/c/en/us/td/docs/iosxml/ios/qosconavd/configuration/xe-

3s/qosconavdxe-3s-book/qosconavdwred-ecn.html

[4] D. Ni, K. Xue, P. Hong, and S. Shen, "Fine-grained forward prediction based dynamic packet scheduling mechanism for multipath TCP in lossy networks," in Proc. 23rd Int. Conf. Comput. Commun. Netw. (ICCCN), Aug. 2014, pp. 17. 
[5] K. Xue et al., "DPSAF: Forward prediction based dynamic packet scheduling and adjusting with feedback for multipath TCP in lossy heterogeneous networks," IEEE Trans. Veh. Technol., vol. 67, no. 2, pp. 1521-1534, Feb. 2018.

[6] M. Handley, J. Padhye, and S. Floyd, TCP Congestion Window Validation, document RFC 2861, IETF, 2000.

[7] M. Honda, Y. Nishida, L. Eggert, P. Sarolahti, and H. Tokuda, "Multipath congestion control for shared bottleneck," in Proc. 7th Int. Workshop Protocols Future, Large-Scale Diverse Netw. Transp., May 2009, pp. 19-24.

[8] S. Barré et al., "Implementation and assessment of modern hostbased multipath solutions," Ph.D. dissertation, Dept. Comput. Sci. Eng., Catholic Univ. Louvain, Louvain-la-Neuve, Belgium, 2011.

[9] Linux MPTCP Kernel. Accessed: Jan. 19, 2020. [Online]. Available: http://www.multipathtcp.org/

[10] F. H. Mirani, N. Boukhatem, and M. A. Tran, "A data-scheduling mechanism for multihomed mobile terminals with disparate link latencies," in Proc. IEEE 72nd Veh. Technol. Conf., Sep. 2010, pp. 1-5.

[11] Q. Peng et al., "Multipath TCP: Analysis, design, and implementation," Networking, IEEE/ACM Transactions on, 2016.

[12] S. R. Pokhrel and M. Mandjes, "Improving Multipath TCP Performance over WiFi and Cellular Networks: an Analytical Approach," IEEE Transactions on Mobile Computing, 2018.

[13] R. Khalili et al., "MPTCP is not pareto-optimal: performance issues and a possible solution," Networking, IEEE/ACM Transactions on, (TON), vol. 21, no. 5, pp. 1651-1665, 2013.

[14] S. R. Pokhrel et al., "Fair Coexistence of Regular and Multipath TCP over Wireless LastMiles," IEEE Transactions on Mobile Computing, vol. 18, no. 3, pp. 574-587, 2019.

[15] S. R. Pokhrel, M. Panda, and H. Vu, "Analytical modeling of multipath TCP over lastmile wireless," Networking, IEEE/ACM Transactions on, p. accepted for publication, 2017.

[16] S. H. Baidya and R. Prakash, "Improving the performance of multipath TCP over heterogeneous paths using slow path adaptation," in ICC. IEEE, 2014, pp. 3222-3227.

[17] S. Ferlin, Ö. Alay, T. Dreibholz, D. A. Hayes, and M. Welzl, "Revisiting congestion control for multipath TCP with shared bottleneck detection," in INFOCOM. IEEE, 2016, pp. 1-9.

[18] M. Pittoni, "Online identification of last-mile throughput bottlenecks on home routers," Ph.D. dissertation, UPMC, 2016.

[19] S. Sundaresan, N. Feamster, and R. Teixeira, "Home network or access link? locating lastmile downstream throughput bottlenecks," in International Conference on Passive and Active Network Measurement. Springer, 2016, pp. 111-123.

[20] "Wireless LAN Medium Access Control (MAC) and Physical Layer (PHY) Specifications, IEEE Std 802.11-2007," June 2007.

[21] S. Ferlin-Oliveira, T. Dreibholz, and O. Alay, "Tackling the challenge of bufferbloat in multi-path transport over heterogeneous wireless networks," in IWQoS, 2014. 
[22] Pokhrel, S.R. and Walid, A., 2021. Learning to harness bandwidth with multipath congestion control and scheduling. IEEE Transactions on Mobile Computing.

[23] Pokhrel, S.R. and Williamson, C., 2018. Modeling compound TCP over WiFi for loT. IEEE/ACM transactions on networking, 26(2), pp.864-878.

[24] Wei, W., Xue, K., Han, J., Wei, D.S. and Hong, P., 2020. Shared bottleneck-based congestion control and packet scheduling for multipath TCP. IEEE/ACM Transactions on Networking, 28(2), pp.653-666.

[25] A. Botta, A. Dainotti, and A. Pescapé, "A tool for the generation of realistic network workload for emerging networking scenarios," Comput. Netw., vol. 56, no. 15, pp. 3531-3547, Oct. 2012.

[26] J. Iyengar, P. Amer, and R. Stewart, "Receive buffer blocking in concurrent multipath transfer," in Proc. IEEE Global Telecommun. Conf., Nov./Dec. 2005, pp. 1-6.

[27] S. R. Pokhrel and A. Walid, "Learning to Harness Bandwidth with Multipath Congestion Control and Scheduling," in IEEE Transactions on Mobile Computing, doi: 10.1109/TMC.2021.3085598.

[28] S. R. Pokhrel and J. Choi, "Low-Delay Scheduling for Internet of Vehicles: Load-Balanced Multipath Communication With FEC," in IEEE Transactions on Communications, vol. 67, no. 12, pp. 8489-8501, Dec. 2019, doi: 10.1109/TCOMM.2019.2941926.

[29] MPTCP NS3 Code. Accessed: Jan. 19, 2020. [Online]. Available: http://code.google.com/p/mptcp-ns3/

[30] Pokhrel, S.R. and Choi, J., 2020. Improving TCP performance over WiFi for internet of vehicles: A federated learning approach. IEEE Transactions on Vehicular Technology, 69(6), pp.6798-6802.

[31] Linux MPTCP Kernel. Accessed: Jan. 19, 2020. [Online]. Available: http://www.multipath-tcp.org/

[32] Pokhrel, Shiva Raj, Youyang Qu, and Longxiang Gao. "Qos-aware personalized privacy with multipath tcp for industrial iot: Analysis and design." IEEE Internet of Things Journal 7.6 (2020): 4849-4861. 